\title{
Galactic Structure and Holography
}

\author{
T. R. Mongan \\ 84 Marin Avenue, Sausalito, CA, USA \\ Email:tmongan@gmail.com
}

How to cite this paper: Mongan, T.R. (2017) Galactic Structure and Holography. Journal of Modern Physics, 8, 2179-2188. https://doi.org/10.4236/jmp.2017.814133

Received: November 18, 2017 Accepted: December 17, 2017 Published: December 20, 2017

Copyright $\odot 2017$ by author and Scientific Research Publishing Inc. This work is licensed under the Creative Commons Attribution International License (CC BY 4.0).

http://creativecommons.org/licenses/by/4.0/

\begin{abstract}
Galactic structure, involving bulk motion of matter conserving mass and angular momentum, is analyzed in a holographic large scale structure model. In isolated galaxies, baryonic matter inhabits dark matter halos with holographic radii determined by total galactic mass. The existence of bulgeless giant spiral galaxies challenges previous models of galaxy formation. In sharp contrast, holographic analysis is consistent with the finding that $15 \%$ of a sample of 15,000 edge-on disk galaxies in the sixth SDSS data release are bulgeless. Holographic analysis also indicates that E7 ellipticals are less than $2 \%$ of elliptical galaxies.
\end{abstract}

\section{Keywords}

Galactic Structure, Holography

\section{Introduction}

This paper does not address the wide range of processes, mechanisms, and mergers generating galactic structures and leading to the myriad galaxy types observed by astronomers. Instead, it identifies the range of isolated galactic structures consistent with conservation of mass and angular momentum in a holographic large scale structure (HLSS) model [1].

Current observations indicate that our universe can be approximated as a closed vacuum-dominated Friedmann universe. Futhermore, "the universe is dominated by an exotic nonbaryonic form of matter largely draped around the galaxies. It approximates an initially low pressure gas of particles that interact only with gravity..." [2]. The holographic principle [3] applied in a vacuum-dominated universe led to an approximate large scale structure model [1] where isolated structures with total mass $M_{g}$ inhabit spherical holographic screens with radius $S=\sqrt{\frac{M_{g}}{0.183 \mathrm{~g} / \mathrm{cm}^{2}}}$ if the Hubble constant $H_{0}=67.8 \mathrm{~km} \cdot \mathrm{sec}^{-1} \cdot \mathrm{Mpc}^{-1}$. 
The HLSS model considers galactic matter density distributions $\rho(r)=\frac{M_{g}}{4 \pi S r^{2}}$, where $r$ is distance from the galactic center. Then, mass within radius $R$ is $(R / S) M_{g}$, and tangential velocity of stars or star clusters in circular orbits within the $1 / r^{2}$ density distribution around the galactic center is $V=\sqrt{G \frac{M_{g}}{S}}$, where $G=6.67 \times 10^{-8} \mathrm{~cm}^{3} \cdot \mathrm{g}^{-1} \cdot \mathrm{sec}^{-2}$.

This analysis treats galaxies as spherical halos of dark matter with radius $S$ and density distribution $\rho(r)=\frac{(1-\alpha) M_{g}}{4 \pi S r^{2}}$ surrounding total baryonic mass $M_{\text {bary }}=\alpha M_{g}$. Cosmological data indicate matter fraction of total energy density of the universe is $\approx 0.31$ and baryonic fraction of total energy desity is $\approx 0.05$, so baryonic fraction of matter is $\alpha \approx 0.05 / 0.31=0.16$ and dark matter fraction is $\alpha-1=0.84$. The analysis uses $\alpha=0.16$, but a distribution of values centered around $\alpha=0.16$ could be used to assess galactic systems formed in parts of the universe with slightly higher or lower ratio of baryons to dark matter. This paper considers galactic structures at redshift $z=0$, but the analysis is readily extended to $z>0$ using results in Ref. [1].

\section{Black Holes in the Galactic Core}

There is no singularity in galactic matter density distribution $\rho(r)=\frac{M_{g}}{4 \pi S r^{2}}$ because mass inside a core radius $C$ at the galactic center is concentrated in a central black hole with mass $M_{C B H}=\frac{C}{S} M_{g}$, where $C$ is the holographic radius of star clusters that can inhabit circular orbits just outside the core without being disrupted and drawn into the central black hole [1]. The mass $M_{S t}$ comprising the dark matter halo with mass $M_{H}$ plus baryonic matter with mass $M_{b a r y}$ is $M_{S t}=M_{g}-M_{C B H}=M_{H}+M_{\text {bary }}=0.84 M_{S t}+0.16 M_{S t}$.

Total angular momentum of a galaxy is estimated using the moment of inertia $I_{g}=\frac{2}{9} M_{g} S^{2}$ of a rotating sphere with galactic holographic radius $S$ and density $\rho(r)=\frac{M_{g}}{4 \pi S r^{2}}$. From the holographic relation $M_{g}=0.183 S^{2}$, total angular momentum of the galaxy is $J_{g}=\left(\frac{2}{9}\right)\left(\frac{M_{g}^{2}}{0.183}\right) \omega_{g}$, where $\omega_{g}$ is galactic angular velocity. Galactic angular velocity $\omega_{g}$ is estimated by considering a mass $m$ fixed on the rotating holographic screen at radius $S$. Radial acceleration $a=-\omega_{g}^{2} S$ of that mass results from gravitational force $F=-\frac{G m M_{g}}{S^{2}}$ attracting it to the centroid of the structure. Then, (correcting a misprint in Ref. [1]) $\omega_{g}^{2}=\frac{G M_{g}}{S^{3}}=\frac{G}{\sqrt{M}}\left(0.183 \mathrm{~g} / \mathrm{cm}^{2}\right)^{\frac{3}{2}}$ and $J_{g}=\frac{2}{9} \frac{G^{0.5}}{\left(0.183 M_{g}\right)^{0.25}} M_{g}^{2}$. In the HLSS 
model [1], average galactic mass at $z=0$ is $8.3 \times 10^{43} \mathrm{~g}$ and average galactic angular momentum is $J_{g}=9 \times 10^{-16} M_{g}^{2}, 15 \%$ higher than Paul Wesson's [4] empirical value $J_{g}=8 \times 10^{-16} M_{g}^{2}$.

Central black hole angular momentum $J_{C B H \max }$ cannot exceed maximum Kerr black hole angular momentum $J_{C B H \max }= \pm\left(\frac{G}{C}\right) M_{K C B H}^{2}= \pm\left(\frac{G}{C}\right)\left(\frac{C}{S}\right)^{2} M_{g}^{2}$, and $\frac{J_{C B H}}{J_{g}} \leq \pm\left[\frac{G}{C}\left(\frac{C}{S}\right)^{2}\right] /\left[\frac{2}{9} \frac{G^{0.5}}{\left(0.183 M_{g}\right)^{0.25}}\right]$. In the HLSS model at $z=0$, galactic mass ranges from $\approx 4 \times 10^{39} \mathrm{~g}$ to $\approx 10^{45} \mathrm{~g}$ and $\left(\frac{C}{S}\right) \leq 1$. So $J_{C B H \max } / J_{g}$ ranges from $\approx 2 \times 10^{-4}$ to $\approx 5 \times 10^{-3}$, and central black hole angular momentum is negligible. Core radius $C$ equals the holographic radius of star cluster sub-elements of the galaxy that can inhabit circular orbits around the galactic center without being disrupted by the central black hole. Maximum HLSS star cluster mass is $4.64 \times 10^{39} \mathrm{~g}$, so $C_{\max }=0.052 \mathrm{kpc}$, and minimum star cluster mass is $2.11 \times 10^{34} \mathrm{~g}$, so $C_{\min }=0.00011 \mathrm{kpc}$.

\section{Spiral Galaxies}

Spiral galaxies predominate in underdense regions of the universe. This analysis treats baryonic matter in isolated spiral galaxies as disk structures with constant tangential velocity $V=\sqrt{G \frac{M_{g}}{S}}$ surrounding a central sphere with constant density.

\section{Conservation of Mass and Angular Momentum}

Baryonic matter outside core radius $C$ around the central black hole consists of a bulge, with mass $M_{B}$ in a hollow sphere with inner radius $C$ and outer radius $B$, and a disk with mass $M_{D S}$. So $M_{\text {bary }}=0.16\left(M_{g}-M_{C B H}\right)=M_{B}+M_{D S}$. Total mass within $B$ and outside the core in the $1 / r^{2}$ density distribution around the galactic center is $(B-C) \frac{M_{g}}{S}$ and tangential velocity at $B$ is

$V_{B}=\sqrt{G \frac{M_{B}}{B}}=V=\sqrt{G \frac{M_{g}}{S}}$. If $M_{B}$, the baryonic part of total mass within $B$, is in a hollow sphere with inner radius $C$ and outer radius $B$,

$$
M_{B}=0.16(B-C) \frac{M_{g}}{S}
$$

Conservation of mass then requires a disk mass

$$
M_{D S}=0.16\left[M_{g}-M_{C B H}-(B-C) \frac{M_{g}}{S}\right]=0.16\left(1-\frac{B}{S}\right) M_{g}
$$

Angular momentum conservation in spiral galaxies requires $J_{g}=J_{C B H}+J_{H}+J_{B}+J_{D S}$, where terms on the right of the equation are 
respectively the angular moment of the central black hole $\left(J_{C B H}\right)$, the dark matter halo $\left(J_{H}\right)$, the central bulge $\left(J_{B}\right)$, and the disk $\left(J_{D S}\right)$. Since $J_{C B H} \leq 5 \times 10^{-3} J_{g}$,

$$
J_{g} \approx J_{H}+J_{B}+J_{D S}
$$

A hollow sphere with density $\rho(r)=\frac{\beta M_{g}}{4 \pi S r^{2}}$, inner radius $C$, and outer radius $R$ has moment of inertia $\frac{2}{9} \beta M_{g}\left(\frac{R^{3}-C^{3}}{R-C}\right)$, so angular momentum $J_{b a r y}$ of galactic baryonic matter comprising the bulge and disk with mass $M_{\text {bary }}=M_{B}+M_{D S}$ is

$$
J_{\text {bary }}=J_{B}+J_{D S}=J_{g}-\frac{2}{9}(0.84) M_{g}\left(\frac{S^{3}-C^{3}}{S-C}\right) \omega_{g}
$$

\section{Bulge Radius and Disk Thickness}

If the hollow sphere of baryonic matter constituting the bulge has constant density $\rho_{B}=\left[0.16(B-C) \frac{M_{g}}{S}\right] /\left[\frac{4}{3} \pi\left(B^{3}-C^{3}\right)\right]$, tangential velocity of baryonic matter in the bulge rises from $V_{C}=\frac{C}{B} V$ at core radius $C$ to $V$ at $B$. The hollow sphere of the bulge with constant density $\rho_{B}$ has moment of inertia $\frac{2}{5} M_{B} \frac{\left(B^{5}-C^{5}\right)}{\left(B^{3}-C^{3}\right)}$. To approximate observed velocity curves, angular velocity of the constant density bulge is found by setting tangential velocity at the edge of the bulge $V_{B}=\omega_{B} B$ equal to disk tangential velocity $V$, so $\omega_{B}=\left(\frac{V}{B}\right)$ and

$$
J_{B}=\frac{2}{5} M_{B}\left(\frac{V}{B}\right) \frac{\left(B^{5}-C^{5}\right)}{\left(B^{3}-C^{3}\right)} .
$$

From Equation (4), disk angular momentum is

$$
J_{D S}=J_{g}-\frac{2}{9}(0.84) M_{g}\left(\frac{S^{3}-C^{3}}{S-C}\right)\left(\frac{V}{S}\right)-\frac{2}{5} M_{B}\left(\frac{B^{5}-C^{5}}{B^{3}-C^{3}}\right)\left(\frac{V}{B}\right) .
$$

Maximum bulge radius $B_{\max }$ occurs when there is no disk and all baryonic mass is bulge mass. From $J_{g}-J_{H}=J_{B_{\max }}$ using $S \gg C$ and $B_{\max }>C$, $\frac{2}{9}(0.16) M_{g} S V \approx \frac{2}{5}(0.16) M_{g} B_{\max } V$ and $B_{\max } \approx \frac{5}{9} S=0.56 S$.

Next, consider a disk extending from $R=B$ to $R=S$ with density $\rho(R, z)=4 \rho_{B} \operatorname{sech}^{2}\left(\frac{z}{z_{0}}\right) \mathrm{e}^{\frac{(R-B)}{K}}$, approximating an isothermal disk with constant scale height $z_{0}$. Setting scale height $z_{0}=H / 8$, the integral $2 \int_{0}^{\frac{H}{2}} 4 \operatorname{sech}^{2}\left(\frac{z}{z_{0}}\right) \mathrm{d} z=8 z_{0} \tanh \left(\frac{H}{2 z_{0}}\right)=H \tanh (4)=0.9993 H$. With constant disk 
height $H$ encompassing $99.9 \%$ of disk matter, disk mass is approximately

$$
M_{D S}=2 \pi H \rho_{B} \int_{B}^{S} \mathrm{e}^{-\frac{(R-B)}{K}} R \mathrm{~d} R=2 \pi H \rho_{B} K\left[(K+B)-(K+S) \mathrm{e}^{-\frac{(S-B)}{K}}\right]
$$

and disk angular momentum is approximately

$$
\begin{aligned}
J_{D S} & =2 \pi H \rho_{B} V \int_{B}^{S} \mathrm{e}^{-\frac{(R-B)}{K}} R^{2} \mathrm{~d} R \\
& =2 \pi H \rho_{B} K V\left[\left(2 K^{2}+2 K B+B^{2}\right)-\left(2 K^{2}+2 K S+S^{2}\right) \mathrm{e}^{-\frac{(S-B)}{K}}\right]
\end{aligned}
$$

For $B=f_{B} S$,

$$
\frac{V M_{D S}}{J_{D S}}=\frac{\left[\left(K+f_{B} S\right)-(K+S) \mathrm{e}^{-\frac{S\left(1-f_{B}\right)}{K}}\right]}{\left[\left(2 K^{2}+2 K f_{B} S+f_{B}^{2} S^{2}\right)-\left(2 K^{2}+2 K S+S^{2}\right) \mathrm{e}^{-\frac{S\left(1-f_{B}\right)}{K}}\right]}
$$

Setting radial scale length $K=x S$, the following equation can be solved for $x$ by trial and error

$$
\frac{S V M_{D S}}{J_{D S}}=\frac{\left[\left(x+f_{B}\right)-(x+1) \mathrm{e}^{-\frac{\left(1-f_{B}\right)}{x}}\right]}{\left[\left(2 x^{2}+2 x f_{B}+f_{B}^{2}\right)-\left(2 x^{2}+2 x+1\right) \mathrm{e}^{-\frac{\left(1-f_{B}\right)}{x}}\right]}
$$

Given $x, H / 2 B$ is found from

$$
\frac{H}{2 B}=\frac{\left(M_{D S} / 2 B\right)}{2 \pi \rho_{B} S^{2} x\left[\left(x+f_{B}\right)-(x+1) \mathrm{e}^{-\frac{\left(1-f_{B}\right)}{x}}\right]}
$$

If sufficient data are available, the model can be extended to consider situations where different stellar populations are in sub-disks with different radial scale lengths and scale heights This is relevant because analysis of the Milky Way [5] found different star populations in sub-disks with scale height inversely related to sub-disk radial scale lengths.

\section{Percentage of Bulgeless Spiral Galaxies}

Kormendy et al. [6] note that bulgeless galaxies with $V>150 \mathrm{~km} / \mathrm{sec}$ $\left(M_{g}>2 \times 10^{44} \mathrm{~g}\right)$ "challenge our picture of galaxy formation by hierarchical clustering." In this analysis, spiral galaxies with mass $M_{g}$ have bulge radii between $C$ and $B_{\max }$. If bulge radius $B^{*}$ approximates the demarcation between spirals with and without a bulge, $\frac{B^{*}-C}{B_{\max }-C}$ is the bulgeless fraction of spiral galaxy configurations consistent with mass and angular momentum conservation. Estimating $B^{*}$ from $\frac{H}{2 B^{*}}=1,6 \%$ to $10 \%$ of galaxies with mass 
ranging from $1 \times 10^{44} \mathrm{~g}$ (with $V=169 \mathrm{~km} / \mathrm{sec}$ ) to $1 \times 10^{45} \mathrm{~g}$ (with $V=300 \mathrm{~km} / \mathrm{sec}$ ) are bulgeless. With scale height $z_{0}=H / 8$, the integral $2 \int_{0}^{\frac{H}{4}} 4 \operatorname{sech}^{2}\left(\frac{z}{z_{0}}\right) \mathrm{d} z=8 z_{0} \tanh \left(\frac{H}{4 z_{0}}\right)=H \tanh (2)=0.964 H$, so a central subdisk of height $H / 2$ contains $96.4 \%$ of disk mass. Estimating $B^{*}$ from $H / 2=2 B^{\star}$, $19 \%$ to $23 \%$ of galaxies with masses between $1 \times 10^{44} \mathrm{~g}$ and $1 \times 10^{45} \mathrm{~g}$ are bulgeless. These estimates bracket the $15 \%$ estimate of bulgeless galaxies found among 15,127 edge-on disk galaxies in the sixth release of SDSS data [7]. So, holographic analysis has no difficulty accounting for bulgeless giant spiral galaxies.

\section{MOND Is Unnecessary}

Dark matter can account for flat velocity curves in spiral galaxies, and observations of the colliding "bullet cluster" galaxies 1E0657-558 provide further evidence for dark matter. To avoid using dark matter to account for flat velocity curves, Modified Newtonian Dynamics (MOND) assumes the law of gravity is different at large distances. Ref. [8] cites the baryonic Tully-Fisher relation and mass discrepancy-acceleration (or $V_{\text {observed }} / V_{\text {Newtonian }}$ ) relation as "challenges for the $\Lambda$ CDM model," and accounts for those relations using MOND with an acceleration threshold $a_{0} \approx 1.2 \times 10^{-8} \mathrm{~cm} / \mathrm{sec}^{2}$. However, the HLSS model [1] within the $\Lambda \mathrm{CDM}$ paradigm readily accounts for the MOND acceleration threshold, baryonic Tully-Fisher relation, and mass discrepancy-acceleration $\left(V_{\text {observed }} / V_{\text {Newtonian }}\right)$ relation. First, radial acceleration at holographic radius $S$ is $a_{S}=\frac{G M_{g}}{S^{2}}=\frac{G\left(0.183 S^{2}\right)}{S^{2}}=0.183 G=1.2 \times 10^{-8} \mathrm{~cm} / \mathrm{sec}^{2}$, equal to MOND acceleration. Second, radial acceleration at radius $R$ due to dark matter is $a_{D M}=\frac{G}{R^{2}}\left(\frac{R}{S}\right) M_{D M}$ and at $R$ sufficiently distant from the galactic center that total baryonic mass of the galaxy $M_{B}=0.16 M_{g}$ can be treated as concentrated at the galactic center, Newtonian radial acceleration from baryonic matter is $a_{B}=\frac{G M_{B}}{R^{2}}$. Radius $R_{\gamma}$ where $a_{D M}=a_{B}$ is found from $\frac{G}{R_{\gamma}^{2}}\left(\frac{R_{\gamma}}{S}\right) M_{D M}=\frac{G M_{B}}{R_{\gamma}^{2}}$. Since $M_{D M}=0.84 M_{g}, R_{\gamma}=0.19 S$, and at that radius $a_{\mathrm{DM}}=a_{B}=5.4 \times 10^{-8} \mathrm{~cm} / \mathrm{sec}^{2}$, near the MOND estimate. Third, tangential velocity $V$ at $R$ relates to radial acceleration $a_{r}$ by $V^{2}=R a_{r}$. So,

$$
\begin{aligned}
& \left(\frac{V_{\text {observed }}}{V_{\text {Newtonian }}}\right)^{2} \approx \frac{R\left(a_{D M}+a_{B}\right)}{R a_{B}} \text {, and } \frac{V_{\text {observed }}}{V_{\text {Newtonian }}}=\sqrt{1+\frac{a_{D M}}{a_{B}}}=\sqrt{1+\frac{R M_{D M}}{S M_{B}}} \text {. When } \\
& R \ll 0.19 S, \frac{V_{\text {observed }}}{V_{\text {Newtonian }}} \approx 1 \text {. Using } \frac{a_{0}}{a_{B}}=\frac{M_{D M}}{0.19 M_{B}}\left(\frac{R}{S}\right)^{2}, \frac{R}{S}=\sqrt{\frac{0.19 M_{B}}{M_{D M}}} \sqrt{\frac{a_{0}}{a_{B}}} \\
& \text { and } \frac{V_{\text {observed }}}{V_{\text {Newtonian }}}=\sqrt{1+\frac{R M_{D M}}{S M_{B}}}=\sqrt{1+\sqrt{\frac{0.19 M_{D M}}{M_{B}}} \sqrt{\frac{a_{0}}{a_{B}}}} \text {. When } a_{B} \ll a_{0}, \frac{a_{0}}{a_{B}} \gg 1
\end{aligned}
$$


and $\frac{V_{\text {observed }}}{V_{\text {Newtonian }}}=\sqrt{1+0.998 \sqrt{\frac{a_{o}}{a_{B}}}} \approx\left(\frac{a_{0}}{a_{B}}\right)^{\frac{1}{4}}$. So, when $a_{B} \ll a_{0},\left(\frac{V_{\text {observed }}}{V_{\text {Newtonian }}}\right)^{4}=\frac{a_{0}}{a_{B}}$ and using $a_{B}=\frac{G M_{B}}{R^{2}}$ and $V_{\text {Newtonian }}=\sqrt{\frac{G M_{B}}{R^{2}}}$, $V_{\text {observed }}^{4}=\left(\frac{G M_{B}}{R^{2}}\right)^{2}\left(\frac{R^{2}}{G M_{B}}\right) a_{0}=G M_{B} a_{0}$, known as the baryonic Tully-Fisher relation. Fourth, if $H_{0}=67.8 \mathrm{~km} \cdot \mathrm{sec}^{-1} \cdot \mathrm{Mpc}^{-1}$, the cosmological constant $\Lambda=1.12 \times 10^{-56} \mathrm{~cm}^{-2}$, and accelerations $\mathrm{cH}_{0}=6.6 \times 10^{-8} \mathrm{~cm} / \mathrm{sec}^{2}$ and $c^{2} \sqrt{\frac{\Lambda}{3}}=5.5 \times 10^{-8} \mathrm{~cm} / \mathrm{sec}^{2}$ are consistent with the acceleration $a_{D M}=a_{B}=5.4 \times 10^{-8} \mathrm{~cm} / \mathrm{sec}^{2}$. So, the MOND hypothesis is unnecessary.

\section{Elliptical Galaxies}

Elliptical galaxies predominate in overdense regions of the universe, and may result from mergers and collisions of progenitor galaxies. Baryonic matter interacts more strongly than dark matter, so collisions between galaxies can produce torques transferring angular momentum from baryonic matter to the composite halo, yielding baryonic ellipsoids with angular velocity less than the angular velocity of the halo and the galaxy as a whole.

This analysis treats elliptical galaxies as constant density ellipsoids within a surrounding spherical isothermal dark matter halo. Dynamical instability of thin galaxies (the firehose instability) limits long axes of ellipsoids to about three times the length of short axes. So elliptical galaxies in this model range from oblate spheroids with radius $A$ along the spin axis equal to one third the radius $R_{\perp}$ perpendicular to the spin axis to prolate spheroids with radius $A$ along the spin axis equal to three times the radius $R_{\perp}$ perpendicular to the spin axis.

In the holographic approach, galaxies are arrangements of bits of information identifying the location of matter in the galaxy. There is no reason for one arrangement of information satisfying conservation of mass and angular momentum to be preferred over another. So, different configurations satisfying conservation of mass and angular momentum with the same total mass should be equally likely.

Baryonic ellipsoids outside the core radius $C$ surrounding the central black hole have baryonic mass $M_{E}=0.16\left(M_{g}-M_{C B H}\right)$ and, since $S \gg C$ and $R_{\perp} \gg C$, the moment of inertia around the spin axis $I_{E} \approx \frac{2}{5} M_{E} R_{\perp}^{2}$. Prolate and oblate spheroidal ellipsoids are analyzed as deformed versions of spheres with radius $R_{o}=\frac{S}{3}$, so the spheroids fit within the holographic screen. Prolate and oblate spheroids with radius $A R_{o}$ along the spin axis have the same mass and angular momentum as spheres with radius $R=R_{o}$. For prolate spheroids capable of avoiding firehose instability, radius along the spin axis ranges from $R_{o}$ to $3 R_{o},(A=1 \rightarrow 3)$ and for corresponding oblate spheroids it ranges 
from $R_{o} / 3$ to $R_{o},\left(A=\frac{1}{3} \rightarrow 1\right)$. Ellipsoid angular velocity depends on how much baryonic angular momentum was transferred to the halo during elliptical galaxy formation.

Hubble class of prolate spheroidal elliptical galaxies with major axes $A$ varying from $R_{o}$ to $3 R_{o}$ and minor axis $B=R_{o}$ is $E=10\left(1-\frac{B}{A}\right)$ rounded to the nearest integer, so limits imposed by firehose instability only allow prolate spheroidal elliptical galaxies of Hubble class $E 0$ to $E 7$. We only see projections of ellipsoids on the plane perpendicular to our line of sight, so actual Hubble class $A E i$ of an elliptical galaxy and its projected (observed) Hubble class Ei are not the same. Prolate elliptical galaxies rotate around their major axis and oblate elliptical galaxies rotate around their minor axis, so results hold for oblate galaxies when $1 / A$ is replaced by $A$, and the projection effect can be understood by considering only prolate spheroids. Table 1 shows $A_{i} R_{o}$ major axis lengths marking transitions between Hubble classes for prolate spheroids, where $A_{i}$ is $A$ projected on the plane perpendicular to the line of sight.

When the angle between rotation axis of prolate elliptical galaxies and the line of sight decreases from $90^{\circ}$ to $0^{\circ}$, the projection $P(\theta)=A \sin \theta$ on the plane perpendicular to the line of sight of the actual $A$ along the axis of rotation gets smaller, so projected shape of the galaxy gets rounder and higher actual Hubble classes appear as lower projected Hubble classes as the axis of rotation gets closer to the line of sight. Hubble class distribution of prolate spheroid shapes projected on the plane perpendicular to the line of sight is found by integrating from $A=1$ to $A=3$ and from $\theta=0$ to $\theta=\frac{\pi}{2}$. As above, this analysis assumes information (matter), in prolate elliptical galaxies capable of avoiding firehose instability with a given mass and angular momentum and corresponding radius $R_{o}$ perpendicular to the rotation axis, is evenly distributed among prolate spheroids with radius $a=A R_{o}$ along the spin axis ranging from $R_{o}$ to $3 R_{o} \quad(A=1 \rightarrow 3)$. Total occupancy of prolate elliptical galaxies with given mass and angular momentum is $\frac{1}{2} \int_{A_{0}=1}^{A_{8}=3} d A=1$. The interval $A E 0$ from $A_{0}$ to $A_{1}$ contributes $\frac{1}{2} \int_{A_{0}=1}^{A_{1}} \mathrm{~d} A=0.026$ to projected Hubble class $E 0$. The interval $A E 1$

Table 1. Major axis lengths $A_{i}$ marking transitions between Hubble classes for prolate spheroids.

\begin{tabular}{cccccccc}
\hline $\begin{array}{c}\text { Hubble class } \\
\text { change }\end{array}$ & $E 0-E 1$ & $E 1-E 2$ & $E 2-E 3$ & $E 3-E 4$ & $E 4-E 5$ & $E 5-E 6$ & $E 6-E 7$ \\
\hline $10\left(1-\frac{1}{A_{t}}\right)$ & 0.5 & 1.5 & 2.5 & 3.5 & 4.5 & 5.5 & 6.5 \\
$\begin{array}{c}\text { Transition } \\
A_{t}\end{array}$ & $A_{1}=1.05$ & $A_{2}=1.18$ & $A_{3}=1.33$ & $A_{4}=1.54$ & $A_{5}=1.82$ & $A_{6}=2.22$ & $A_{4}=2.86$ \\
\hline
\end{tabular}


Table 2. Projected Hubble class Ei of elliptical galaxies versus actual Hubble class AEi of elliptical galaxies.

\begin{tabular}{cccccccccc}
\hline & $E 0$ & $E 1$ & $E 2$ & $E 3$ & $E 4$ & $E 5$ & $E 6$ & $E 7$ & Total \\
\hline$A E 7$ & 0.005 & 0.001 & 0.002 & 0.003 & 0.005 & 0.009 & 0.032 & 0.015 & 0.071 \\
$A E 6$ & 0.029 & 0.008 & 0.011 & 0.018 & 0.032 & 0.076 & 0.143 & 0 & 0.317 \\
$A E 5$ & 0.030 & 0.008 & 0.013 & 0.021 & 0.048 & 0.082 & 0 & 0 & 0.202 \\
$A E 4$ & 0.031 & 0.009 & 0.015 & 0.032 & 0.052 & 0 & 0 & 0 & 0.140 \\
$A E 3$ & 0.033 & 0.011 & 0.023 & 0.036 & 0 & 0 & 0 & 0 & 0.103 \\
$A E 2$ & 0.036 & 0.017 & 0.026 & 0 & 0 & 0 & 0 & 0 & 0.078 \\
$A E 1$ & 0.043 & 0.019 & 0 & 0 & 0 & 0 & 0 & 0 & 0.062 \\
$A E 0$ & 0.026 & 0 & 0 & 0 & 0 & 0 & 0 & 0 & 0.026 \\
Projected & 0.233 & 0.074 & 0.089 & 0.110 & 0.137 & 0.168 & 0.174 & 0.015 & \\
\hline
\end{tabular}

from $A_{1}$ to $A_{2}$ contributes $\frac{1}{2} \int_{A_{1}}^{A_{2}} \mathrm{~d} A=0.062$ to projected Hubble classes $E 0$ and $E 1$, broken down as $\frac{1}{2} \int_{A_{1}}^{A_{2}} \mathrm{~d} A\left[\int_{0}^{\theta_{1}(A)} \sin \theta \mathrm{d} \theta+\int_{\theta_{1}(A)}^{\pi / 2} \sin \theta \mathrm{d} \theta\right]$ where

$\theta_{i}(A)=\sin ^{-1}\left(\frac{A_{i}}{A}\right)$, the first term in the integral contributes to projected Hubble class $E 0$, and the second term contributes to projected Hubble class $E 1$. Similarly, the interval $A E i$ from $A_{i}$ to $A_{1+1}$ contributes to all projected Hubble classes from $E 0$ to $E$ i, broken down as

$\frac{1}{2} \int_{A_{i}}^{A_{i+1}} \mathrm{~d} A\left[\int_{0}^{\theta_{1}(A)} \sin \theta \mathrm{d} \theta+\sum_{j=i}^{i-1} \int_{\theta_{j}(A)}^{\theta_{j+1}(A)} \sin \theta \mathrm{d} \theta+\int_{\theta_{i}(A)}^{\pi / 2} \sin \theta \mathrm{d} \theta\right]$ where the first term contributes to projected Hubble class $E 0$, the last term contributes to projected Hubble class $E i$, and intervening terms contribute to projected Hubble classes from $E 1$ to $E(i-1)$. Use $\int_{\theta_{J}(A)}^{\theta_{j+1}(A)} \sin \theta \mathrm{d} \theta=\left(\cos \theta_{j}(A)-\cos \theta_{j+1}(A)\right)$ and $\cos \left[\sin ^{-1}\left(\frac{A_{j}}{A}\right)\right]=\sqrt{1-\left(\frac{A_{j}}{A}\right)^{2}}$ to get

$\int_{\theta_{j}(A)}^{\theta_{j+1}(A)} \mathrm{d} \theta \sin \theta=\frac{1}{A}\left(\sqrt{A^{2}-A_{j}^{2}}-\sqrt{A^{2}-A_{j+1}^{2}}\right)$, and $\int \mathrm{d} A\left(\frac{1}{A}\right) \sqrt{A^{2}-\xi^{2}}=\sqrt{A^{2}-\xi^{2}}+\xi \tan ^{-1}\left(\frac{\xi}{\sqrt{A^{2}-\xi^{2}}}\right)$. This results in Table 2,

listing fractional distribution of projected Hubble class of elliptical galaxies. The result suggests Hubble class E7 galaxies comprise $<2 \%$ of elliptical galaxies.

\section{References}

[1] Mongan, T.R. (2013) Journal of Modern Physics, 4, 50-54. https://doi.org/10.4236/jmp.2013.47A1006

[2] Peebles, P.J.E. (2017) How the Nonbaryonic Dark Matter Theory Grew. Princeton University, Princeton.

[3] Bousso, R. (2002) Reviews of Modern Physics, 74, 825. 
https://doi.org/10.1103/RevModPhys.74.825

[4] Wesson, P. (1981) Physical Review D, 23, 1730.

https://doi.org/10.1103/PhysRevD.23.1730

[5] Bovy, J., et al. (2012) The Astrophysical Journal, 753, 148.

[6] Kormendy, J., et al. (2010) The Astrophysical Journal, 723, 54.

[7] Kautsch, S.J. (2009) Astronomische Nachrichten, 330, 100-106.

[8] Famaey, B. and Mc Gaugh, S. (2012) Living Reviews in Relativity, 15, 10. 